\title{
Evaluation of GeneXpert MTB/RIF Assay for Detection of Pulmonary Tuberculosis on Sputum Samples
}

\author{
Sajjad Ahmad1, Sumera Afzal2, Aman Ullah³ and Anwar Sheed 4
}

\begin{abstract}
Objective: To analyse the diagnostic performance of MTB/RIF assay for the diagnosis of pulmonary tuberculosis and detection of rifampicin resistance using sputum samples.

Study Design: Observational cross-sectional study.

Place and Duration of Study: Provincial TB Reference Laboratory (PTRL), Hayatabad Medical Complex, Peshawar, Pakistan, from January to October 2015.

Methodology: A total of 268 participants were consecutively enrolled in the study after meeting the inclusion criteria. Their sputum samples were collected and processed by $\mathrm{N}$-acetyl-L-cysteine-sodium hydroxide (NALC-NaOH) method and GeneXpert MTB/RIF assay.

Results: This study determined the overall sensitivity and specificity of MTB/RIF assay, it was $92.4 \%$ (86/93) and $97.1 \%$ (138/142), respectively. The sensitivity was $98.4 \%$ (60/61) in culture proven smear positive samples, whilst sensitivity in culture proven smear negative samples was $93.7 \%$ (30/32), using culture as reference standard.

Conclusion: GeneXpert MTB/RIF assay could greatly improve early diagnosis of PTB in smear negative cases as well as multidrug resistant tuberculosis.
\end{abstract}

Key Words: GeneXpert, MTB/RIF assay, Pulmonary tuberculosis, Multidrug resistant tuberculosis.

\section{INTRODUCTION}

Tuberculosis (TB) remains the deadliest health problem around the Globe, with 9.0 million new cases of TB, whilst 1.5 million deaths in 2013, which rank TB as a second single killer infectious disease after human immunodeficiency virus (HIV) throughout the world.1,2

TB also stands a major health concern in Pakistan with 370,000 to 650,000 incident cases and 45,000 to 170,000 deaths per 100,000 populations in 2013. Pakistan stands fifth among 22 TB high burden countries and at fourth position among 27 countries flourished with multidrug resistant tuberculosis (MDR-TB). ${ }^{2}$ There are 36,000 new TB cases develope every year in Khyber Pakhtunkhwa (KPK). ${ }^{3}$ There is a pressing need of early diagnosis with prompt treatment in order to reduce morbidity, mortality and transmission of TB.

Sputum smear microscopy is the most commonly used test for the diagnosis of TB, having $68 \%$ rate of case

1 Institute of Basic Medical Sciences, Khyber Medical University, Peshawar, Pakistan

2 Center of Biotechnology and Microbiology, University of Peshawar, Pakistan

3 Institute of Paramedical Sciences, Khyber Medical University, Peshawar, Pakistan

4 Provincial Reference Laboratory for Tuberculosis, Hayatabad Medical Complex, Peshawar, Pakistan

Correspondence: Mr. Aman Ullah, Institute of Paramedical Sciences, Khyber Medical Sciences, Peshawar, Pakistan E-mail: khurramthalwi@hotmail.com

Received: August 12, 2017; Accepted: August 21, 2018 detection. 4 In 2010, 2.0 million smear negative cases were found among 5.8 million notified cases worldwide, and revealed great instance of TB misdiagnosis. ${ }^{5}$

Methods based on culturing of tubercle bacilli are the gold standard for the diagnosis of TB, but due to requirements of sophisticated infrastructure and times in weeks and months, it hampers the early diagnosis of TB, 6 and can lead to delayed initiation of required treatment which increases the possibility of transmission and development of drug resistant due to the commencement of improper therapy. ${ }^{7}$

To address the urgent need of prompt diagnosis, several molecular assays were designed based on nucleic acid amplification including line probe assay (Genotype MTBDRplus assay) and Gene Xpert MTB/RIF assay. MTBDRplus line probe assay (LPA) was endorsed by World Health Organization (WHO) in 2008 for the early detection of mycobacterium tuberculosis complex (MTBC) and it also serves for the rapid screening of multidrug resistant tuberculosis (MDR-TB) in acid fast bacilli (AFB) smear positive sputum, by detection of most frequent genetic mutation conferring resistance to rifampicin (RIF) and isoniazid (INH). ${ }^{8}$ Unfortunately LPA requires spacious infrastructure and cannot be performed outside the reference laboratories. ${ }^{9}$

WHO implemented MTB/RIF assay in 2010 for the earlier diagnosis of TB and detection of RIF resistance simultaneously by targeting the 81 base pair region in rpoB gene, determining the RIF resistance. It is a fully automated real time PCR performed in a single use cartridge and gives result within 2 hours. 10 RIF 
resistance can act as a surrogate marker for the detection of MDR-TB because it has been reported in more than $95 \%$ strains of MDR-TB. 11 It is directly used on respiratory specimen of TB suspect unlike LPA, which can only be performed on smear positive sputum or culture from a smear negative sample.4

The given study was conducted to evaluate the MTB/RIF assay for the diagnostic performance in clinically suspected pulmonary tuberculosis (PTB) at Provincial TB Reference Laboratory (PTRL), Peshawar, Pakistan.

\section{METHODOLOGY}

The given work was performed at PTRL, Hayatabad Medical Complex, after the approval of Ethical Committee of PTRL. A total of 268 participants were consecutively enrolled in the study from January to October 2015, referred by their physician as a TB suspect from different healthcare facilities of Peshawar, Pakistan. A sputum sample was collected from each participant in a clean, dry, wide mouth plastic bottle after filling a standardized questionnaire for demographic data and signing of Informed Consent by participants or legal guardians for use of sputum in TB diagnostic research.

For the identification of MTBC a direct smear was prepared from fresh sputum and examined using light microscopy after Ziehl-Neelsen (ZN) staining, according to the standard protocol. Sputum was decontaminated by $\mathrm{N}$-acetyl-L-cysteine-sodium hydroxide (NALC-NaOH) method and 2 to 3 drops of the sediment were inoculated on two Lowenstein Jensen (LJ) slants. All the slopes of $\mathrm{LJ}$ medium were incubated at $37^{\circ} \mathrm{C}$ and examined within 2-4 days for the demonstration of fast growing mycobacteria and contaminant organism. The culture was declared positive, when colonies of characteristic morphology were grown and AFB were determined using $\mathrm{ZN}$ stain. Each isolate was biochemically identified by using p-Nitrobenzoic Acid (PNB) susceptibility, catalase test, and nitrate reduction test. ${ }^{12}$ All the isolates were further confirmed by MTBDRplus line probe assay and also determined the resistance against INH and RIF.

The sample reagent of MTB/RIF assay was added to the sediment remain in the falcon tube after decontamination and the cap was tightly screwed, afterward it was incubated at room temperature for 15 minutes with vigorous shaking after each 5 minutes interval. The liquefied sample was transferred to the open port of MTB/RIF cartridge with a sterile dropper. Finally the MTB/RIF cartridge was loaded in the chamber of GeneXpert instrument and result was recorded within 2 hours according to the instruction of manufacturer. ${ }^{13}$

For statistical analysis, descriptive data were presented in number and percentages. The overall sensitivity and specificity of MTB/RIF assay was compared with culture results as a reference standard. While sensitivity of MTB/RIF assay was also compared with tuberculosis culture in both categories of smear positive culture positive and smear negative culture positive cases.

\section{RESULTS}

A total of 268 sputum samples were collected from the same numbers of participants who met inclusion criterion, Amongst 268 specimens, 27 were contaminated, five were Xpert invalid/error, two were non-tuberculosis mycobacterium (NTM) and one was LPA undermining result. After the exclusion of 35 samples, only 233 sputum samples were subjected to the analysis.

Out of 233 samples, 61 (26.2\%) were found positive for AFB on direct smear microscopy, while 172 (73.8\%) specimens gave negative results for the detection of AFB. MTB/RIF assay determined MTBC in 86 (36.9\%) pulmonary specimens while failed to detect MTBC in the remaining $147(63.1 \%)$ samples, of the $86(36.9 \%)$ positive cases of MTB/RIF assay, 60 (69.7\%) specimens were smear-positive, whilst $26(30.3 \%)$ samples were smear-negative.

Among the 233 specimens subjected to the conventional LJ culture, $93(39.9 \%)$ were able to grow and gave positive result for MTBC, while 140 (60.1\%) samples were declared negative after 8 weeks of incubation. Sixty-one $(65.5 \%)$ specimens were smear positive and $32(34.5 \%)$ samples were smear negative among 93 $(39.9 \%)$ culture positive specimens.

LPA demonstrated 92 (98.9\%) MTBC and displayed uninterpretable result in case of one $(1.1 \%)$ culture positive specimen.

MTB/RIF assay revealed RIF resistance in 10 (10.7\%) sputum samples, while LPA also determined the same but nine (90\%) were MDR-TB and one (10\%) was only RIF resistant. In 93 (39.9\%) confirmed cases of TB, the MTB/RIF assay showed $92.4 \%$ sensitivity and $97.1 \%$

Table I: Comparison of geneXpert MTB/RIF-positive, MTB culture-positive results with smear results.

\begin{tabular}{|c|c|c|c|c|}
\hline & \multicolumn{3}{|c|}{ Sensitivity } & \multirow{2}{*}{$\begin{array}{c}\text { Specificity } \\
\text { No tuberculosis }\end{array}$} \\
\hline & All culture+ & $\begin{array}{c}\text { Smear+ } \\
\text { and culture+ }\end{array}$ & $\begin{array}{c}\text { Smear- } \\
\text { and culture+ }\end{array}$ & \\
\hline Xpert + & 86 & 60 & 26 & 4 \\
\hline \multirow[t]{2}{*}{ Xpert - } & 7 & 1 & 6 & 136 \\
\hline & $\begin{array}{c}86 / 86+7 \times 100 \\
=92.4 \%\end{array}$ & $\begin{array}{c}60 / 60+1 \times 100 \\
=98.4 \%\end{array}$ & $\begin{array}{c}26 / 26+6 \times 100 \\
=81.25 \%\end{array}$ & $\begin{array}{c}136 / 136+4 \times 100 \\
=97.1 \%\end{array}$ \\
\hline
\end{tabular}


specificity using solid culture as a reference standard. When the culture positive specimens were stratified by microscopy results, the MTB/RIF assays was $98.4 \%$ and $81.25 \%$ sensitive for smear positive and smear negative cases, respectively. Table I shows the analytical performance of Xpert MTB/RIF assay compared to other methods.

\section{DISCUSSION}

It was an endeavour to analyse the diagnostic performance of MTB/RIF assay on sputum samples from TB suspects in TB endemic area, using culture as a reference standard. This study indicated that overall sensitivity and specificity of MTB/RIF assay were $92.4 \%$ and $97.1 \%$, respectively. The findings of the study pertinent to the sensitivity of MTB/RIF assay validate the $97.6 \%$ and $95.3 \%$ earlier reported sensitivity of Boehme et al. and Pinyopornpanish et al., respectively. ${ }^{14,6}$ The slight difference in the sensitivity may be due to the number of sputum specimens collected per participant, because here one specimen was collected, while others had collected three specimens. The specificity of MTB/RIF assay in the current study is almost in line with the $99 \%$ specificity shown by Boehme et al., which extends the previous knowledge.

The findings were stratifiedd on the basis of microscopy results, the sensitivity of the MTB/RIF was $98.4 \%$ among both culture and smear positive specimens; while in case of culture positive and smear negative specimens, the sensitivity was $81.25 \%$. Previously, the sensitivity of the smear positive specimens has been cited from $98-100 \%$ and smear negative specimens were found to be sensitive from $74.2-77.7 \% .15,12$ The present findings are concordant with the results of earlier studies in regard of culture proven smear positive TB. The given work determined higher sensitivity of MTB/RIF assay in case of smear negative TB because they used pellet of the decontaminated samples instead of direct expectorated sputum, which could be the likely reason of this higher sensitivity. Boehme et al. determined $85.1 \%$ sensitivity for smear negative TB by using pellet of the decontaminated sputum. ${ }^{14}$ Furthermore, this study used only sputum while other studies include all possible pulmonary samples.

The current research demonstrated seven cases which were positive on culture and negative on Xpert MTB/RIF assay amongst smear negative specimens. The possible explanation for this contradiction could be the difference in detection of analytical limit because culture can detect as low as $10-100 \mathrm{CFU} / \mathrm{ml}$, while MTB/RIF assay detect $131 \mathrm{CFU} / \mathrm{ml} .{ }^{11}$

Interestingly Xpert MTB/RIF assay detected MTBC in four sputum samples which were declared negative by microscopy and culture. These specimens were not repeated on culture and were also not run on any other molecular technique to validate the results of MTB/RIF assay. Rachow et al. deemed the similar results as true positive on the basis of clinical TB. ${ }^{16}$

MTB/RIF assay declared 10 specimens positive for RIF resistances which were also positive by MTBDRplus assay but nine (90\%) of these specimens were MDR-TB and one $(10 \%)$ specimen was monoresistant, as only resistant to RIF. Though comparison with MTBDRplus assay encouraged the results of MTB/RIF assay, but still further studies are needed using drug susceptibility testing (DST) as a reference standard.

Limitations of the present study are the high rate (10\%) of contamination in solid culture and unavailability of any technique for the confirmation of positive results of MTB/RIF assay in specimens, which were declared negative by both culture and smear. DST was not perforemed, which is also the limitation of the current research because we could not compare the results of RIF resistance by MTB/RIF assay with reference standard.

\section{CONCLUSION}

MTB/RIF assay is recommended for the detection of pulmonary TB, particularly in smear negative specimens. In addition to this, it is also indicated that the MTB/RIF assay can also accelerate the early detection of MDR-TB in smear positive specimens.

\section{REFERENCES}

1. Maru M, Mariam SH, Airgecho T, Gadissa E, Aseffa A. Prevalence of tuberculosis, drug susceptibility testing, and genotyping of mycobacterial isolates from pulmonary tuberculosis patients in dessie, Ethiopia. Tuberc Res Treat 2015; 2015:1-10.

2. World Health Organization. Global tuberculosis control: WHO report 2014. World Health Organization 2014; Geneva, Switzerland.

3. Javaid A, Ghafoor A, Rab A, Basit A, Ullah Z, Ali S, et al. Primary drug resistance to antituberculous drugs in NWFP Pakistan. J Pak Med Assoc 2008; 58:437-40.

4. Aurin TH, Munshi SK, Kamal SM, Rahman MM, Hossain MS, Marma T, et al. Molecular approaches for detection of the multidrug resistant tuberculosis (MDR-TB) in Bangladesh. PloS One 2014; 9:e99810.

5. World Health Organization. Global tuberculosis control 2010. World Health Organization. 2010; Geneva, Switzerland.

6. Pinyopornpanish K, Chaiwarith R, Pantip C, Keawvichit R, Wongworapat $\mathrm{K}$, Khamnoi $\mathrm{P}$, et al. Comparison of xpert MTB/RIF assay and the conventional sputum microscopy in detecting mycobacterium tuberculosisin Northern Thailand. Tuberc Res Treatment 2015; 2015:571782.

7. Espinal MA, Kim SJ, Suarez PG, Kam KM, Khomenko AG, Migliori GB, et al. Standard short-course chemotherapy for drug-resistant tuberculosis: treatment outcomes in 6 countries. JAMA 2000; 283:2537-45.

8. Wilson ML. Recent advances in the laboratory detection of mycobacterium tuberculosis complex and drug resistance. Clin Infect Dis 2011; 52:1350-5. 
9. Boehme CC, Nicol MP, Nabeta P, Michael JS, Gotuzzo E, Tahirli $\mathrm{R}$, et al. Feasibility, diagnostic accuracy, and effectiveness of decentralised use of the Xpert MTB/RIF test for diagnosis of tuberculosis and multidrug resistance: a multicentre implementation study. Lancet 2011; 377:14951505.

10. Shrestha P, Arjyal A, Caws M, Prajapati KG, Karkey A, Dongol S, et al. The application of geneXpert MTB/RIF for smearnegative TB diagnosis as a fee-paying service at a South Asian General Hospital. Tuberc Res Treatment 2015; 2015:1-6.

11. Marlowe EM, Novak-Weekley SM, Cumpio J, Sharp SE, Momeny MA, Babst A, et al. Evaluation of the cepheid xpert MTB/RIF assay for direct detection of mycobacterium tuberculosis complex in respiratory specimens. J Clin Microbiol 2011; 49:1621-3.

12. Sharma SK, Kohli M, Yadav RN, Chaubey J, Bhasin D, Sreenivas $\mathrm{V}$, et al. Evaluating the diagnostic accuracy of Xpert
MTB/RIF assay in pulmonary tuberculosis. PloS One 2015; 10:e0141011.

13. World Health Organization. Rapid implementation of the Xpert MTB/RIF diagnostic test: technical and operational 'how to' practical considerations. World Health Organization. 2011; Geneva, Switzerland.

14. Boehme CC, Nabeta P, Hillemann D, Nicol MP, Shenai S, Krapp F, et al. Rapid molecular detection of tuberculosis and rifampin resistance. N Engl J Med 2010; 363:1005-15.

15. Zeka AN, Tasbakan S, Cavusoglu C. Evaluation of the GeneXpert MTB/RIF assay for rapid diagnosis of tuberculosis and detection of rifampin resistance in pulmonary and extrapulmonary specimens. J Clin Microbiol 2011; 49:4138-41.

16. Rachow A, Zumla A, Heinrich N, Rojas-Ponce G, Mtafya B, Reither $\mathrm{K}$, et al. Rapid and accurate detection of Mycobacterium tuberculosis in sputum samples by Cepheid Xpert MTB/RIF assay-a clinical validation study. PloS One 2011; 6:e20458. 